\title{
The Impact of COVID-19 on Spanish Health Professionals: A Description of Physical and Psychological Effects
}

\author{
Mònica Cunill ${ }^{1}$, Maria Aymerich ${ }^{1}$, Bernat-Carles Serdà ${ }^{2, *}$ and Josefina Patiño-Masó ${ }^{3}$ \\ ${ }^{1}$ Faculty of Education and Psychology, Quality of Life Research Institute, University of Girona, Girona, 17071, Spain \\ ${ }^{2}$ Health and Healthcare Research Group and Girona Biomedical Research Institute (IDIBGI), Health Sciences Department, \\ University of Girona, Girona, 17071, Spain \\ ${ }^{3}$ Nursing Department, Quality of Life Research Institute, University of Girona, Girona, 17071, Spain \\ *Corresponding Author: Bernat-Carles Serdà. Email: bernat.serda@udg.edu \\ Received: 19 May 2020; Accepted: 04 July 2020
}

\begin{abstract}
Aim: To describe the physical and psychological symptoms in healthcare workers caring for COVID-19 patients. Methods: Cross-sectional descriptive study design. A sample of 1,452 participants was collected. Sociodemographic data were recorded. Symptoms of anxiety were screened with Generalized Anxiety Disorder (GAD-7), symptoms of depression were measured with the Patient Health Questionnaire (PHQ-9), and finally physical symptoms were measured using the Patient Health Questionnaire (PHQ-15). Percentages, means and standard deviations, the one-way and two-way ANOVA test, the Chi square test and Pearson's correlation coefficient were all calculated. The level of significance was $(p<0.05)$. Results: Medium levels of anxiety (range, 5-9) and depression (range, 5-9) were observed, as well as the existence of physical symptoms (5-30). Nursing staff presented higher scores for anxiety than medical staff (Bonferroni test $=-1.68 ; p<0.001$ ). Significant differences were also found for depression ( $\mathrm{F}=9.8 ; p<0.001)$. Nursing staff and assistant nursing staff presented higher scores than medical professionals (Post hoc test $=-2.11 ; p<0.001$ and Post hoc test $=-1.53 ; p<0.001$, respectively). Significant differences were found according to gender in all variables referring to emotional distress: anxiety ( $\mathrm{t}$ student $=-6.492 ; p<0.001)$, depression ( $\mathrm{t}$ student $=-4.703 ; p<0.001)$ and physical symptoms ( $\mathrm{t}$ student $=-9.015 ; p<0.001$ ). Female healthcare workers displayed anxiety, depression and physical symptoms more frequently than their male counterparts. Analysing gender differences within each professional activity, no significant differences were found using the two-way ANOVA test $(\mathrm{F}=1.52$; $p=0.109$ ). Anxiety and depression correlated with physical symptoms ( $\mathrm{rxy}=$ 0.604; $p<0.001$ ) when applying Pearson's correlation coefficient. Conclusions: The study confirms the psychological impact and manifestation of physical symptoms in healthcare workers during the COVID-19 outbreak, highlighting the need to monitor symptoms and provide effective psychological intervention to prevent adverse effects on mental health such as post-traumatic stress disorder and burnout.
\end{abstract}

Keywords: COVID-19; healthcare worker; physical symptoms; psychological symptoms; anxiety; depression; emotional distress

This work is licensed under a Creative Commons Attribution 4.0 International License, which permits unrestricted use, distribution, and reproduction in any medium, provided the original work is properly cited. 


\section{Introduction}

Following the first diagnosed case of COVID-19 in Wuhan (China) in December 2019 and the declaration of a coronavirus pandemic by the United Nations in March 2020 [1], over 2,081,969 cases of COVID-19 had been diagnosed in more than two hundred countries and territories by 16 April 2020, with a death toll of 138,487. Among the most affected countries have been the United States, followed by Spain, Italy, Germany, France and China [2]. The rapid spread of the pandemic and its high incidence have forced governments to reorganize their healthcare systems to guarantee the best care and respond to the risk of a collapse associated with the saturation of intensive care units due to the high number of COVID-19 patient admissions.

In Spain, as of April 16, 2020, the total number of confirmed infected cases was 182,816, of whom 76,752 had required hospitalization and 7,916 admission to intensive care. The total number of deceased was 19,1303 [3]. After the state of alarm was decreed on March 14 [4], four exceptional measures were taken to strengthen the National Health System and contain the health crisis caused by the pandemic. New hospitals were created, services reorganized, and retired professionals and/or senior medical students recruited, while the shortage of personal protective equipment (PPE) and safety equipment necessary to prevent contagions among health personnel was also addressed. For all of the above reasons, health professionals, and especially those who work in hospitals and care for people with confirmed or suspected COVID-19, are under extreme pressure.

According to research made available from China, health professionals are vulnerable to a high risk of infection and mental health problems [5]. Indeed, a recent study [6] evaluating mental health outcomes among healthcare workers $(\mathrm{n}=1257)$ treating patients with COVID-19 in China, specifically in Wuhan (60.8\%), Hubei $(20.8 \%)$ and outside Hubei province $(18.8 \%)$, found that a considerable proportion reported symptoms of depression (50.4\%), anxiety (44.6\%) and distress (71.5\%). Guo et al. [7] have also examined the psychological impact of the COVID-19 outbreak on Chinese medical staff $(\mathrm{n}=11,118)$, with around $4.99 \%$ and $13.47 \%$ reporting high levels of anxiety and depression symptoms, respectively.

In Spain, 20.4\% of COVID-19 cases reported to the National Centre for Epidemiology have been by health workers, this percentage being higher among women than men $(28.1 \%$ vs. $11.3 \%) ; 10 \%$ of these professionals have been hospitalized during the first outbreak of the pandemic [8].

Some studies carried out on previous epidemics have observed that healthcare personnel who work in highly stressful and life-threatening environments are prone to experiencing emotional distress $[9,10]$ and developing different mental disorders [11-13]. Under these conditions, some of the most stressful factors that can be experienced by health professionals in the workplace at the psychological level and that favour the appearance of anxiety and/or depression disorders are: increased work overload, extension of the working day, the feeling of making a superhuman effort, not having adequate personal protective equipment and/or being afraid or feeling very scared [14,15]. Age, sex, professional activity performed, the type of health centre and/or unit where they work, years of work experience and/or proximity to the patient may all increase the onset of symptoms [16]. In addition, the use of personal protective equipment may add fatigue, discomfort (heat, limitation of spontaneous movements), mild bodily disorders (dehydration, frequent urination and/or rashes on the skin), physical and/or social isolation and/or increased constant monitoring of infection control measures and/or safety procedures [17]. Psychiatric anxiety and morbidity generally decrease over time, although relapses may appear, especially for those health professionals with more vulnerable pre-existing mental health [11].

Based on an analysis of 61 studies that evaluate the impact of epidemics on health professionals' mental health, Ricci-Cabello et al. [18] observed high levels of anxiety, followed by depression, acute stress disorder and post-traumatic stress disorder (PTSD) and burnout both during and after outbreaks. Another study, which 
analysed the immediate psychological consequences in health professionals with a high risk of exposure during the MERS-CoV outbreak in Saudi Arabia, observed that these are mainly fatigue, insomnia, concern about one's own health and fear of contagion [19]. The two studies concurred that these psychological consequences not only have a lasting effect on healthcare providers' mental health, but also interfere with the capacity to provide care and the quality associated with it in the short and long term.

A recent systematic review and meta-analysis [20] examining the effects of the COVID-19 outbreak on healthcare workers' mental health revealed higher rates of anxiety and depression among females, while nursing staff exhibited a higher prevalence of anxiety and depression than to doctors. Although these results may be partly biased by the fact that nurses are mostly female, they could also be attributed to the greater risk of exposure to COVID-19 patients, given that nurses spend more time on the ward and provide patients with direct care.

According to Collado et al. [21], although the risk of health professionals presenting physical and/or psychological symptoms as part of an adaptive response to the stressful situation to which they are subjected increases during the pandemic period, such symptoms need to be identified promptly so as to provide professionals with the necessary resources and avoid the situation becoming chronic and/or having greater effects.

This study aimed to describe the physical and psychological symptoms displayed by health professionals who care for patients infected with COVID-19 during the first phase of the pandemic.

Hypothesis 1. The incidence of anxiety, depression, and physical symptoms among Spanish healthcare professionals will be significantly high.

Hypothesis 2. Nursing assistants and nurses will display significantly more distress than doctors.

Hypothesis 3. Gender differences will be observed: women will display significantly higher levels of anxiety, depression and physical symptoms than men.

Hypothesis 4. A higher level of emotional distress is positively related to more physical symptoms.

\section{Method}

\subsection{Design}

Descriptive cross-sectional comparative study.

\subsection{Participants}

The study population comprises health professionals caring for patients in hospitals, primary care centres and/or socio-health care centres throughout Spain. Non-probability sampling was carried out for convenience.

The final sample of health professionals who participated in the present study $(\mathrm{N}=1,452)$ comprised $82.9 \%(1,204)$ women. As for age, 53.9\% (783) of participants were between 30 and 50 years old, 32\% (464) over 50 and $14 \%$ (204) under 30 . With regard to origin, $87 \%(1,263)$ of the participants were from Catalonia and the rest other autonomous regions in Spain. In relation to their occupation, $44.7 \%$ were nursing staff (649), 26.4\% (383) medical professionals, 11.5\% (167) nursing assistants, 1\% (14) hospital porters and the rest $16.4 \%$ (204) from other professions (pharmacists, physical therapists, residents and students of health sciences). A total of $73.3 \%(1,064)$ reported having over 10 years' professional experience and $73.1 \%(1,061)$ gave their employment status as permanent. The demographic characteristics of the participants are presented in Tab. 1.

\subsection{Procedure}

The data were collected by means of an online questionnaire from April 4 to 10, during the peak period of contagion and death from the coronavirus [3]. The questionnaire was disseminated via social networks (WhatsApp, Instagram, Telegram and email) following the criteria posited by Di Lonardo et al. [22]. The 
Table 1: Percentage and frequency of health professionals' demographic characteristics

\begin{tabular}{|c|c|c|c|}
\hline Baseline characteristics & & $n$ & $\%$ \\
\hline \multicolumn{4}{|l|}{ Gender } \\
\hline & Male & 248 & 17.1 \\
\hline & Female & 1,204 & 82.9 \\
\hline \multicolumn{4}{|l|}{ Age } \\
\hline & $\leq 30$ years & 204 & 14 \\
\hline & 31-39 years & 341 & 23.5 \\
\hline & $40-49$ years & 442 & 30.5 \\
\hline & $\geq 50$ years & 465 & 32 \\
\hline \multicolumn{4}{|l|}{ Professional activity } \\
\hline & Nursing assistant & 167 & 11.5 \\
\hline & Hospital porter & 14 & 1 \\
\hline & Nurse & 649 & 44.7 \\
\hline & Doctor & 383 & 26.4 \\
\hline & Other professionals & 203 & 16.4 \\
\hline \multicolumn{4}{|l|}{ Work unit } \\
\hline & Primary care/Health Centre & 216 & 14.9 \\
\hline & Emergency room/Intensive care & 287 & 15.9 \\
\hline & $\begin{array}{l}\text { Socio-sanitary residence/Geriatric } \\
\text { Hospital }\end{array}$ & 70 & 4.8 \\
\hline & COVID-19 patient centre & 110 & 7.6 \\
\hline & Hospitalization & 702 & 55.8 \\
\hline & Other & 67 & 4.6 \\
\hline \multicolumn{4}{|l|}{ Years of work experience } \\
\hline & $<1$ year & 33 & 2.3 \\
\hline & From 1 to 3 years & 127 & 8.7 \\
\hline & From 4 to 9 years & 228 & 15.7 \\
\hline & $\geq 10$ years & 1,064 & 73.3 \\
\hline \multicolumn{4}{|l|}{ Professional status } \\
\hline & Temporary contract & 289 & 19.9 \\
\hline & Permanent contract & 1,061 & 73.1 \\
\hline & Other & 292 & 7 \\
\hline \multicolumn{4}{|l|}{ Hours of work per shift } \\
\hline & Between 7 and 8 hours & 633 & 43.6 \\
\hline & From 8 to 10 hours & 215 & 14.8 \\
\hline & From 10 to 12 hours & 362 & 24.9 \\
\hline & $>12$ hours & 242 & 16.7 \\
\hline
\end{tabular}


relevant ethical principles were followed to ensure compliance with the processing of participants' personal data, their public freedom and fundamental rights according to the Spanish Data Protection Act and the Declaration of Helsinki [23,24].

Participants were invited to complete the questionnaire using the online "Google Forms" tool, which allows invitations to be sent online through social media. Before answering the online questionnaire, participants were informed about its duration (five minutes), the identity of the researchers and the aim of the study. It was also clearly stated that only doctors, nurses, nursing assistants, hospital porters and/or healthcare support staff could answer the questionnaire, together with health science students. No incentive was offered to participate in the study. All participants voluntarily signed an informed consent document before responding.

The use of the Internet and the online survey greatly facilitated data collection during one of the initial and most stressful periods of the pandemic for healthcare professionals.

Although the results derived from the convenience sample used in this study cannot be extrapolated to all Spanish health professionals, a very high number of responses was received, and they are therefore very useful for generating ideas and hypotheses of interest regarding the study population.

\subsection{Measures}

An ad hoc questionnaire was used to collect participants' sociodemographic data (sex, age group and geographical location), job characteristics in their professional activity (nursing assistant, hospital porter, nurse, doctor, other professionals), work unit (primary care/healthcare centre, emergency room/intensive care, home/geriatric hospital, COVID-19 patient centre, hospital, other), years of work experience (less than 1 year, from 1 to 3 years, from 4 to 9 years and 10 years or more), employment status (temporary or permanent contract, other) and hours of work per shift (from 7 to 8 hours, 8 to 10 hours, 10 to 12 hours, more than 12 hours) during the covid-19 outbreak, and the degree to which the health crisis had affected their and/or their families' professional health activity, if applicable. A five-point Likert scale $(1=$ not at all; $5=$ a lot) was used to capture the extent to which healthcare workers felt the following: work overload, emotional burnout from work, helpless/scared, they could not do their job to the best of their ability, continuously overstrained, and they might have to leave the profession for the first time. The questionnaire included two items for those professionals who were not working at the time of administration (feeling guilty, feeling useless about not being able to do their job). In addition, it asked about the sufficient availability of self-protection measures, professionals' level of discomfort with these and the extent to which they allowed professionals to adequately carry out their professional activity. More specifically, using a Likert-type scale $(0=$ not at all; $10=$ a lot $)$ they were asked how concerned they were about getting COVID-19, getting sick and their health worsening, that their life was in danger, or that the health of their relatives was being affected by the situation that they were going through. They were also asked about various protection measures used by health workers. Specifically, whether these measures were considered sufficient or insufficient. Using a Likert-type scale $(1=$ not at all uncomfortable; 5 = very uncomfortable), they were asked about the discomfort generated by the use of surgical masks, FFP2-FFP3 masks, gloves, long-sleeved waterproof gowns, special frame glasses and full-face shields. The same type of response scale was used to ask about the degree of difficulty generated by personal protective equipment in terms of physical discomfort, communication difficulties with patients, difficulties in recognizing others, loneliness and communication difficulties with co-workers.

The study also identified symptoms of anxiety and depression, as well as the somatization associated with the two mentioned disorders, using the Spanish-validated versions of the Generalized Anxiety Disorder scale (GAD-7) (range, 0-21) [25]; the 9-item Patient Health Questionnaire (PHQ-9; range, 027) [26] and the 15-item Patient Health Questionnaire (PHQ-15; range 0-30) 2020 [27]. The GAD-7 
version adapted into Spanish displayed very promising metric properties, the Cronbach's alpha being excellent (0.936). All items showed a high item-total correlation (higher than 0.68 ), with a test-retest correlation of 0.844 and an intra-class correlation coefficient of 0.926 (95\% confidence interval of $0.881-0.958$ ). All items obtained the highest score for the congruence index in the GAD objective domain, with scores ranging from 0.50 to the maximum possible value of 1 . The discriminant validity was excellent, all items yielding significant differences $(p<0.001)$ in all cases when quartiles 1 and 4 were compared [25]. In the Spanish version of the PHQ-9, there was good agreement between diagnoses and those of an independent mental health professional (for the diagnosis of any PHQ disorder, kappa $=0.74$; overall accuracy, 88\%; sensitivity, 87\%; specificity, 88\%) in the Spanish version, which was similar to the original English version of the PHQ-9 in primary care patients [26]. The Spanish PHQ-15 questionnaire showed acceptable internal reliability (Cronbach's Alpha Coefficient $=0.78$ ) and adequate validity, with correlations with the MADRS scale between moderate and high $(r=0.3-0.7)$ and differences between groups of patients [27].

The internal consistency of the questionnaires used in our sample was as follows: in the GAD-7, the Cronbach's Alpha Coefficient $=0.89$, in the PHQ-9, the Cronbach's Alpha Coefficient $=0.87$ and in the PHQ-15, the Cronbach's Alpha Coefficient $=0.86$.

The total scores from these evaluative measures were interpreted as follows: GAD-7, normal anxiety (0-4), medium anxiety (5-9), moderate anxiety (10-14) and severe anxiety (15-21); PHQ-9, absence of depression (0-4), mild depression (5-9), moderate depression (10-14) and severe depression (15-21); and PHQ-15, no physical symptoms (0-4), existence of physical symptoms (5-30). The cut-off points for diagnosing anxiety, depression or physical symptoms were 10, 7 and 4, respectively. These measurements were based on values established by the scientific literature [18-20].

\subsection{Data Analysis}

A total of 1,481 questionnaires were collected, and a data cleaning procedure was applied prior to analysis. Raw data cleaning consisted in identifying, correcting or removing unclean data. The aim was to make sure that the data were accurate, consistent and clear before conducting the analysis, as unclean data can ultimately distort the results. The procedure involved running a preliminary analysis and crosschecking unexpected results against the data in the questionnaires. In the present study, it was decided that questionnaires with over $30 \%$ of the items unanswered would be considered invalid and they would consequently be eliminated from the dataset. Any invalid or apparently nonsensical values, duplicated rows of data, rows of data for unidentifiable individuals and rows of blank data were eliminated. This cleaning procedure offered both quality control and external quality. On completion of the cleaning procedure, $29(2 \%)$ questionnaires were discarded and 1,452 questionnaires considered valid for analysis.

Different statistical inference techniques were used. Percentages and absolute frequencies were used to describe the sociodemographic characteristics and physical symptoms of the sample. Means with standard deviations (SD) were used to describe the intensity of symptoms anxiety, depression and physical symptoms. Student's $t$-test ( $t$ student) was used to determine the differences between the means of anxiety and depression by gender. A one-way ANOVA was conducted to verify differences by professional activity, while a two-way ANOVA was used to test for gender differences within each professional activity.

Finally, Pearson's correlation coefficient and Chi square tests were applied to analyse the relationship between anxiety, depression and physical symptoms. A significance level of 0.05 was selected. Version 26 of the SPSS program was used to analyse the data.

\subsection{Results}

\subsubsection{The Impact of COVID-19 on Healthworkers' Concerns and Feelings}

With regard to infection, $49.9 \%$ (725) of participants reported that they had not been infected with COVID-19 and 41.1\% (597) did not know. When asked if they had a family member infected with the virus living with them, $73.4 \%(1,066)$ answered that they did not and 19.8\% (287) did not know. 
As for their concerns, $89.7 \%(1,302)$ of health professionals reported being concerned about being infected with the virus (mean $=7.44$; $\mathrm{SD}=2.32)$, 92.2\% $(1,339)$ about getting sick and their health worsening (mean $=7.94$; $\mathrm{SD}=2.27)$ and $88.4 \%(1,282)$ that their life was in danger (mean $=7.70 ; \mathrm{SD}=$ 2.48). A higher percentage, $99 \%(1,426)$, expressed concern about the health of their relatives (mean $=$ 9.32; $\mathrm{SD}=1.26)$, while $68.7 \%$ (983) felt totally helpless and/or scared.

As for work, $88.1 \%(1,260)$ felt they were very overworked and $77.1 \%(1,101)$ reported feeling emotionally burned out at work, while $77.5 \%(1,104)$ said that they could not do their job to the best of their ability. In addition, 46.7\% (653) of professionals said they would like to be somewhere else and/or be someone else right now. Equally, 63.4\% (904) felt that they were continually overstrained and 20.6\% (296) had considered leaving the profession for the first time when all this ends.

Of those professionals in the sample who were not working at the time $(\mathrm{N}=130), 50.2 \%$ felt guilty and $45.8 \%$ useless for not being able to do their job.

With regard to protection measures, $71 \%(1,031)$ of the professionals thought they were insufficient (mean $=2.77$; $\mathrm{SD}=1.15)$ and $83.5 \%(1,213)$ considered the personal protective equipment used to be affecting the quality of patient care.

Tab. 2 presents data regarding the degree of discomfort caused by the different protection measures used to deal with COVID-19 and the main difficulties associated with these. The health professionals surveyed perceived the personal protective equipment to be uncomfortable and widely reported communication difficulties with patients and among co-workers.

Table 2: Perception of discomfort with main protection measures

\begin{tabular}{llll}
\hline & \multicolumn{3}{c}{ Responses } \\
\cline { 2 - 4 } & $\mathrm{N}$ total & $\mathrm{n}$ & $\%$ \\
\hline Perception of discomfort & & & \\
Surgical masks & 1,443 & 1,176 & 81.5 \\
FFP2-FFP3 masks & 1,303 & 1,137 & 87.3 \\
Gloves & 1,425 & 428 & 30 \\
Long-sleeved waterproof gown & 1,252 & 850 & 67.9 \\
Special frame glasses & 1,196 & 1,059 & 88.5 \\
Use of full-face shield & 1,068 & 815 & 76.3 \\
Difficulties associated with personal protective equipment & & & \\
Physical discomfort & 1,247 & 1,147 & 92 \\
Communication difficulties with the patient & 1,278 & 1,185 & 92.7 \\
Difficulties in recognizing others & 1,283 & 1,150 & 89.6 \\
Loneliness & 1,273 & 904 & 71 \\
Communication difficulties with co-workers & 1,276 & 1,090 & 85.4 \\
\hline
\end{tabular}

\subsubsection{Anxiety, Depression and Physical Symptoms}

Medium levels of anxiety (range, 5-9) and depression (range, 5-9) were observed, together with the existence of physical symptoms (5-30). In addition, there was a high dispersion between scores (see Tab. 3). 
Table 3: Means and standard deviations (SD) of the anxiety, depression and physical symptoms variables

\begin{tabular}{llll}
\hline & GAD-7 & PHQ-9 & PHQ-15 \\
\hline $\mathrm{M}$ & 8.78 & 8.62 & 24.27 \\
$\mathrm{SD}$ & $(4.91)$ & $(5.55)$ & $(5.75)$ \\
$\mathrm{n}$ & 1,403 & 1,308 & 1,154 \\
\hline \multicolumn{2}{l}{ Note: $\mathrm{M}=$ mean; SD $=$ Standard Derivations } & &
\end{tabular}

Tab. 4 shows the frequency and percentage of responses referring to physical symptoms (PHQ-15) presented by health professionals in the study. In this regard, 63.4\% (732) presented what can be considered relevant symptoms. The most frequently reported self-reported problems were: headache, back and spine pain, fatigue, and insomnia. Of all participants, 63.2\% (918) reported that these problems had created difficulties for them with regard to doing their work, doing activities at home and/or housework, or in their relationships with others.

Table 4: Percentage and frequency of physical symptoms presented by health professionals

\begin{tabular}{llll}
\hline Physical problems & & \multicolumn{2}{c}{ Responses } \\
\cline { 3 - 4 } & $\mathrm{N}$ total & $\mathrm{n}$ & $\%$ \\
\hline Headache & 1,432 & 1140 & 79.6 \\
Fatigue or low energy & 1,426 & 1059 & 74.2 \\
Insomnia & 1,428 & 1059 & 74.1 \\
Arm, leg or joint pain & 1,417 & 900 & 63.5 \\
Constipation, loose stools or diarrhea & 1,421 & 777 & 54.7 \\
Palpitation & 1,429 & 674 & 47.3 \\
Stomach ache & 1,426 & 674 & 47.2 \\
Nausea, gas or indigestion & 1,420 & 592 & 41.7 \\
Dyspnea or Shortness of breath & 1,420 & 573 & 40.3 \\
Chest pain & 1,417 & 528 & 37.2 \\
Dizziness & 1,417 & 428 & 30.2 \\
Pain or problems in the sexual act & 1,310 & 216 & 16.5 \\
Back pain & 1,421 & 110 & 78.1 \\
Fainting & 1,398 & 32 & 2.3 \\
\hline
\end{tabular}

\subsubsection{Distress by Health Professionals' Job}

Tab. 5 presents the means and standard deviations (SD) for the anxiety and depression variables in health professionals according to their job. A one-way ANOVA revealed significant differences in levels of anxiety according to the type of work professionals do $(\mathrm{F}=10,075 ; p<0.001)$.

A multiple comparison test revealed that nursing staff presented higher scores than medical professionals (Bonferroni test $=-1.68 ; p<0.001)$. Significant differences were also found for depression $(\mathrm{F}=9.8 ; p<0.001)$, nursing staff and nursing assistant staff presenting higher scores than medical professionals (Bonferroni test $=$ $-2.11 ; p<0.001$ and Bonferroni test $=-1.53 ; p<0.001$, respectively) (Tab. 5). 
Table 5: Means and Standard Deviations (SD) of the anxiety and depression variables in health professionals according to their work activity

\begin{tabular}{|c|c|c|c|c|c|c|c|c|}
\hline \multirow[t]{2}{*}{ Measure } & \multicolumn{2}{|c|}{$\begin{array}{c}\text { Doctor } \\
\mathrm{n}=367\end{array}$} & \multicolumn{2}{|c|}{$\begin{array}{c}\text { Nurse } \\
\mathrm{n}=626\end{array}$} & \multicolumn{2}{|c|}{$\begin{array}{l}\text { Nursing assistant } \\
\mathrm{n}=161\end{array}$} & \multicolumn{2}{|c|}{$\begin{array}{l}\text { Hospital porter } \\
\mathrm{n}=14\end{array}$} \\
\hline & M & SD & M & SD & $\mathrm{M}$ & SD & $\mathrm{M}$ & SD \\
\hline GAD-7 & 7.92 & 4.90 & 9.60 & 4.94 & 9.02 & 4.87 & 8.78 & 4.72 \\
\hline PHQ-9 & 7.45 & 5.36 & 9.56 & 5.75 & 8.98 & 5.30 & 9.23 & 5.44 \\
\hline
\end{tabular}

\subsubsection{Anxiety, Depression and Physical Symptoms by Gender}

Significant differences were found by gender in all variables referring to emotional distress: anxiety ( $\mathrm{t}$ student $=-6.492 ; p<0.001$ ), depression ( $\mathrm{t}$ student $=-4.703 ; p<0.001)$ and physical symptoms (t student $=-9.015 ; p<$ 0.001). Also, women displayed anxiety, depression and physical symptoms more frequently than men (Tab. 6).

Table 6: Means and standard deviations (SD) in the gender variable according to scores for anxiety, depression and physical symptoms

\begin{tabular}{|c|c|c|c|c|c|}
\hline \multirow[t]{2}{*}{ Gender } & \multicolumn{2}{|c|}{ Men } & \multicolumn{2}{|c|}{ Women } & \multirow{2}{*}{$\frac{\text { TOTAL }}{n}$} \\
\hline & $\mathrm{M}$ & SD & M & SD & \\
\hline GAD-7 & 6.92 & 4.72 & 9.16 & 4.86 & 1,402 \\
\hline PHQ-9 & 7.04 & 5.51 & 8.95 & 5.50 & 1,308 \\
\hline PHQ-15 & 21.23 & 4.88 & 24.99 & 5.71 & 1,154 \\
\hline
\end{tabular}

\subsubsection{Relationship between Emotional Distress and Physical Symptoms}

In this regard, those professionals who presented signs of anxiety had more physical symptoms than those who did not, and the same is true of those presenting signs of depression (Tab. 7).

Finally applying the Pearson correlation coefficient anxiety states correlated significantly with depressive states $(\mathrm{rxy}=0.734 ; p<0.001)$ and physical symptoms ( $\mathrm{rxy}=0.604 ; p<0.001)$. Equally, depressive states correlated significantly with the existence of physical symptoms ( $\mathrm{rxy}=0.678 ; p<0.001)$.

Table 7: Percentages, frequencies and $\mathrm{X}^{2}$ test related to the relationship between anxiety, depression and physical symptoms

\begin{tabular}{|c|c|c|c|c|c|}
\hline \multirow[b]{3}{*}{ Anxiety (GAD) $\mathrm{N}=1,117$} & \multicolumn{4}{|c|}{ Physical symptoms (PHQ-15) } & \\
\hline & \multicolumn{2}{|c|}{ Clinical symptoms } & \multicolumn{2}{|c|}{ No clinical symptoms } & \\
\hline & $\%$ & $\mathrm{n}$ & $\%$ & $\mathrm{n}$ & \\
\hline Presence of anxiety & 88.4 & 372 & 11.6 & 49 & \\
\hline \multirow[t]{2}{*}{ No anxiety } & 49 & 342 & 51 & 355 & \\
\hline & & & & & $\mathrm{X}_{2}=176.09 * *$ \\
\hline \multicolumn{6}{|c|}{ Depression (PHQ-9) $\mathrm{N}=1,054$} \\
\hline Presence of depression & 86.1 & 451 & 13.9 & 73 & \\
\hline \multirow[t]{2}{*}{ No depression } & 40.9 & 217 & 59.1 & 315 & \\
\hline & & & & & $\mathrm{X}_{2}=231.16 * *$ \\
\hline
\end{tabular}

$* * p<0.001$ 


\section{Discussion}

This study confirms the psychological impact and manifestation of physical symptoms in healthcare professionals during the exercising of their professional activity in the first phase of the COVID-19 pandemic in Spain. These results coincide with those of other previous works $[5,6,28]$.

The sociodemographic profile of the participants corresponded mainly to women aged between 30 and 50. Medical professionals and nurses represent $71.1 \%$ of the sample. More than half of the respondents work in hospitalization units, have a permanent employment contract and proven professional experience of over 10 years.

In this first phase of analysis, $41 \%$ of professionals reported not knowing whether they had the disease or not. This is mainly attributed to the lack of diagnostic tests in Spain during this period of the epidemic [29,30]. Also, the shortage of protective material to ensure that at-risk medical staff could carry out their healthcare work safely constituted an additional factor in their feeling mostly defenceless and/or scared, while at the same time generating great concern about the possibility of becoming infected and/or infecting their relatives and/or loved ones and the risk of their health worsening, endangering their lives and that of those close to them [6,29]. Presumably, this uncertainty and perceived risk of becoming ill might change if contingent support is provided by the mental health service, adequate protective material is provided, and the polymerase chain reaction (PCR) test and screening based on the serological test are applied to health professionals [31,32].

In accordance with the findings of other studies, a high prevalence of emotional distress was recorded among health professionals in this study [10], medium anxiety and depression levels being observed in all participants [27]. Moreover, physical symptoms were identified.

By professional profile, greater depression is confirmed in the subgroup of nursing and nursing assistant professionals compared to doctors during infectious epidemics, while nurses return greater anxiety scores than doctors. One possible explanation for this result is that nurses and nursing assistants are directly and actively involved in patient care, so they experience a higher risk of contagion. Worries and concerns about the perceived risk of being infected or the possibility of infecting their family and/or friends correlate with psychological distress. On the other hand, having sufficient information was associated with a lower degree of concern in this study, with doctors having better access to medical information and mostly regarding themselves as sufficiently informed [10].

Symptoms of anxiety and depression are more frequent among females than males in the group of healthcare workers. Other research carried out during the COVID-19 pandemic $[6,15,20,33]$ found that being a woman carries a higher risk for experiencing symptoms of anxiety and/or depression. There are also inter-gender differences in the presentation of physical symptoms. These data are consistent with those of other studies $[14,15]$, which show that in situations of professional stress, women in healthcare show greater physical symptoms than their male counterparts. Possibly, the long working hours in this phase of the pandemic, combined with the direct contact with infected patients and/or strict isolation measures, which on many occasions involve distancing from their loved ones to avoid contagion, add additional pressure on women, especially if they have children and/or elderly relatives at home. This should therefore be taken into account in preventive programmes and/or actions aimed at protecting the mental health of the highest risk subgroup, in this case, women health workers.

The findings support evidence of a positive relationship between emotional distress and physical symptoms. Among the registered physical symptoms reported by health professionals in this study, it is worth highlighting headache, back pain, fatigue and insomnia. Rajkumar [34] has observed a greater number and intensity of physical symptoms among the subgroup of health professionals than the rest of the general population during the pandemic period. Given the above, the authors feel the hypothesis of a psychosomatic relationship should be validated in future studies $[35,36]$. 
Together these results suggest a risk that some health professionals may be suffering from burnout syndrome [37]. The observed predisposing factors to burnout confirmed by other studies correspond to: a) work overload with continued physical, mental and emotional overstrain; b) the longest and most intense working hours; c) isolation and discomfort, as well as difficulty communicating due to the use of protective equipment; and d) a perception of not being able to carry out professional duties effectively, all of which affects the quality of care provided $[38,39]$. The results of the systematic review compiled by Hall et al. [40] confirm that decreased well-being among health professionals associated with moderate and high levels of burnout results in medical errors that condition the patient's safety. Finally, it should be noted that one fifth of the sample reported considering leaving the profession when the pandemic ends. According to Koinis et al. [14] a stressful work environment that is perceived as dangerous plays a determining role in health professionals' decision to stay in the same workplace or leave. The feeling of vulnerability and loss of control, concern about their own health and that of their family members, as well as the numerous and rapid changes in working conditions, intensify the perception of danger [6]. Based on this finding, a psycho-educational programme in emergency preparedness training should be developed to help health professionals deal with anxiety and depression. This should include proactive approaches to manage work fatigue and workload stress. The health authorities should also plan the advance availability of psychological support programmes.

Based on the results observed in this study, the prevalence of emotional distress - including anxiety and depression - and physical symptoms is expected to increase significantly among health professionals once the acute phase of the COVID-19 pandemic is over.

By way of conclusion, in order to not underestimate the registered symptoms and to establish effective prevention measures, it is recommended that mental health monitoring be implemented to ensure early identification of those health professionals at risk and assess the need for the most appropriate treatment in the short or long term, thereby reducing the risk of PTSD or burnout. On the other hand, a greater number of studies is needed in our country and internationally in order to research the association between COVID-19 and the physical and psychological effects observed in health professionals.

\subsection{Implications}

A prevalence of emotional distress, including anxiety, depression and physical symptoms, was found among Spanish health workers during the first peak of the COVID-19 outbreak, in addition to other risk factors that impact on their health. It is therefore of the utmost importance that care be provided for the mental health of professionals health workers. Health professionals are in need of mental health protection and adequate working conditions, including the provision of necessary and sufficient medical protective equipment and access to PCR tests, disinfection protocols, adequate rest, and recovery programmes aimed at empowering the coping and resilience process to improve psychological well-being.

A tailored protocol for healthcare workers must therefore be designed and implemented, providing guidelines and training on mental health risks. Early psychological interventions targeting vulnerable groups, such as women in healthcare, may be beneficial in this respect. In order to minimize face-to-face interactions, electronic devices and technological support could initially be used. Early healthcare intervention could start between healthcare workers and later continue with an online psychological service for rehabilitation [41]. Some strategies that would ensure psychological support might include story sharing with counselling services. If anxiety and depression are not addressed through early psychological intervention, the risk of PTSD and other health implications increase significantly. Being proactive could play a pivotal role in substantially lowering the risk of developing long-term mental health illnesses during and after COVID-19, and also in future potential outbreaks. 


\subsection{Future Directions}

This study confirms a positive relationship between psychological distress and physical symptoms. Future studies should confirm this overlap between mental and somatic disorders and lend support to a unified construct of functional somatic disorder [42].

\subsection{Limitations}

This study provides an overview of the emotional impact the coronavirus is having on the surveyed health professionals. However, since it involved convenience sampling and voluntary participation, the results obtained are not representative and cannot be extrapolated to all Spanish healthcare professionals.

Furthermore, it should be noted that there has been an uneven incidence of the pandemic in the different autonomous regions, meaning that it could have a different impact and affect health workers in different ways in the various regions.

The data provided refer to responses obtained over six days at the peak of the pandemic. A subsequent longitudinal follow-up of the sample would be necessary to observe what changes have occurred as a result of the epidemic continuing and the amount of both staff and material resources available to deal with it.

This study does not consider pre-existing clinical situations or previous mental illnesses of the participating health professionals, which could make them more vulnerable to suffering a greater psychological impact in this context.

Funding Statement: The author(s) received no specific funding for this study.

Conflicts of Interest: The authors declare that they have no conflicts of interest to report regarding the present study.

\section{References}

1. World Health Organization (2020). Naming the coronavirus disease (COVID-19) and the virus that causes it 2020. https://www.who.int/emergencies/diseases/novel-coronavirus-2019/technical-guidance/naming-the-coronavirusdisease-(covid-2019)-and-the-virus-that-causes-it.

2. Johns Hopkins University (2020). Coronavirus COVID-19 Dashboard by the center for systems science and Engineering (CSSE). Johns Hopkins University. Baltimore, Maryland. https://coronavirus.jhu.edu/map.html.

3. European Centre for Disease Prevention and Control (2020). Communicable disease threats report (CDTR). Week 16, 12-18 https://www.ecdc.europa.eu/sites/default/files/documents/Communicable-disease-threats-report-18-apr2020-PUBLIC.pdf.

4. Ministry of the Presidency (2020). Relations with the Courts and Democratic Report, Royal Decree 463/2020, of March 14, declaring the state of alarm for management of the health crisis caused by COVID-19. Official State Bulletin, 67, 25390-25400, https://www.boe.es/eli/es/rd/2020/04/10/487.

5. Xiang, Y. T., Yang, Y., Li, W., Zhang, L., Zhang, Q. et al. (2020). Timely mental health care for the 2019 novel coronavirus outbreak is urgently needed. The Lancet Psychiatry, 7(3), 228-229. DOI 10.1016/S2215-0366(20)30046-8.

6. Lai, J., Ma, S., Wang, Y., Cai, Z., Hu, J. et al. (2020). Factors associated with mental health outcomes among health care workers exposed to coronavirus disease 2019. JAMA Network Open, 3(3), e203976. DOI 10.1001/ jamanetworkopen.2020.3976.

7. Guo, J., Liao, L., Wang, B., Li, X., Guo, L. et al. (2020). Psychological effects of COVID-19 on hospital staff: a national cross-sectional survey of China mainland. The Lancet Psychiatry, SSRN Electron. J. https://ssrn.com/ abstract $=3550050$.

8. Ministry of Science and Innovation, Carlos III Health Institute, National Network of Epidemiological Surveillance (2020). Report on the situation of COVID-19 in Spain. Report COVID no 25 of April, 2020. Madrid: Ministry of Science and Innovation. https:/www.isciii.es/QueHacemos/Servicios/VigilanciaSaludPublicaRENAVE/EnfermedadesTransmisibles/ Paginas/InformesCOVID-19.aspx.

9. Greenberg, N., Wessely, S., Wykes, T. (2015). Potential mental health consequences for workers in the Ebola regions of West Africa-A lesson for all challenging environments. Journal of Mental Health, 24(1), 1-3. DOI 10.3109/09638237.2014.1000676. 
10. Goulia, P., Mantas, C., Dimitroula, D., Mantis, D., Hyphantis, T. (2010). General hospital staff worries, perceived sufficiency of information and associated psychological distress during the A/H1N1 influenza pandemic. $B M C$ Infectious Diseases, 10(1), 938. DOI 10.1186/1471-2334-10-322.

11. Vyas, K. J., Delaney, E. M., Webb-Murphy, J. A., Johnston, S. L. (2016). Psychological impact of deploying in support of the US response to Ebola: a systematic review and meta-analysis of past outbreaks. Military Medicine, 181(11), e1515-e1531. DOI 10.7205/MILMED-D-15-00473.

12. Bitanihirwe, B. K. (2016). Monitoring and managing mental health in the wake of Ebola. Ann Ist Super Sanità, 52 (3), 320-322. DOI 10.4415/ANN_16_03_0 Commentary.

13. Aiello, A., Khayein, M. Y., Raja, S., Peladeu, N., Romano, D. et al. (2011). Resilience training for hospital workers in anticipation of an influenza pandemic. Journal of Continuing Education in the Health Professions, 31(1), 15-20. DOI 10.1002/chp.20096.

14. Koinis, A., Giannou, V., Drantaki, V., Angelaina, S., Stratou, E. et al. (2015). The impact of healthcare workers job environment on their mental-emotional health. Coping strategies: the case of a local general hospital. Health Psychology Research, 3(1), 12-17. DOI 10.4081/hpr.2015.1984.

15. Liu, Z., Han, B., Jiang, R., Huang, Y., Ma, C. et al. (2020). Mental health status of doctors and nurses during COVID-19 epidemic in China, THELANCET, https://ssrn.com/abstract=3551329.

16. Bohlken, J., Schömig, F., Lemke, M. R., Pumberger, M., Riedel-Heller, S. G. (2020). COVID-19-pandemie: belastungen des medizinischen personals [COVID-19 pandemic: stress experience of healthcare workers-a short current review]. Psychiatrische Praxis, 47(4), 190-197. DOI 10.1055/a-1159-5551.

17. Cao, J., Wei, J., Zhu, H., Duan, Y., Geng, W. et al. (2020). A study of basic needs and psychological wellbeing of medical workers in the fever clinic of a tertiary general hospital in beijing during the COVID-19 outbreak. Psychotherapy and Psychosomatics, 89(4), 252-254. DOI 10.1159/000507453.

18. Ricci-Cabello, I., Meneses-Echavez, J. F., Serrano-Ripoll, M. J., Fraile- Navarro, D., Fiol de Roque, M. A. et al. (2020). Impact of viral epidemic outbreaks on mental healthcare workers: a rapid systematic review. Medrxiv Journal, 1-92. https://www.medrxiv.org/content/10.1101/2020.04.02.20048892v1.full.pdf.

19. Alsubaie, S., Hani Temsah, M., Al-Eyadhy, A. A., Gossady, I., Hasan, G. M. et al. (2019). Middle East Respiratory Syndrome Coronavirus epidemic impact on healthcare workers' risk perceptions, work and personal lives. The Journal of Infection in Developing Countries, 13(10), 920-926. DOI 10.3855/jidc.11753.

20. Pappa, S., Ntella, V., Giannakas, T., Giannakoulis, V. G., Papoutsi, E. et al. (2020). Prevalence of depression, anxiety, and insomnia among healthcare workers during the COVID-19 pandemic: a systematic review and meta-analysis. Brain, Behavior, and Immunity, [Preprint]. DOI 10.1016/j.bbi.2020.05.026.

21. Collado, B., Torre, Y. (2015). Attitudes toward the risk prevention in health professionals in case of epidemiological alert. Medicina y Seguridad del Trabajo, 61(239), 254-272.

22. Di Lonardo, A., Donfrancesco, C., Iannucci, L., Gargiulo, L., Palmieri, L. C. et al. (2017). Ad hoc surveys: how to measure and report quality methods. Epidemiology Biostatistics and Public Health, 14(3), e12732-1-e12732-9. DOI $10.2427 / 12732$.

23. ECIJA (2018). Organic law $3 / 2018$ of December 5 on the protection of personal data and guarantee of digital rights. BOE Technology, Spain. 119788-119857, https://ecija.com/en/sala-de-prensa/organic-law-3-2018-ofdecember-5-protection-of-personal-data-and-guarantee-of-digital-rights/.

24. World Medical Association (2013). Declaration of Helsinki: ethical principles for medical research involving human subject. JAMA, 310(20), 2191-2194. DOI 10.1001/jama.2013.281053.

25. García-Campayo, J., Zamorano, E., Ruiz, M. A., Pardo, A., Pérez-Páramo, M. et al. (2010). Cultural adaptation into Spanish of the generalized anxiety disorder-7 (GAD-7) scale as a screening tool. Health and Quality of Life Outcomes, 8(1), 8. DOI 10.1186/1477-7525-8-8.

26. Diez-Quevedo, C., Rangil, T., Sanchez-Planell, L., Kroene, K., Spitzer, R. (2001). Validation and utility of the patient health questionnaire in diagnosing mental disorders in 1003 general hospital Spanish inpatients. Psychosomatic Medicine, 63(4), 679-686. DOI 10.1097/00006842-200107000-00021. 
27. Ros, S., Comas, A., Garcia-Garcia, M. (2010). Validation of the Spanish version of the PHQ-15 questionnaire for the evaluation of physical symptoms in patients with depression and/or anxiety disorders: DEPRE-SOMA study. Actas Españolas de Psiquiatria, 38(6), 345-357.

28. Montemurro, N. (2020). The emotional impact of COVID-19: from medical staff to common people. Brain, Behavior, and Immunity, 87, 23-24. DOI 10.1016/j.bbi.2020.03.032.

29. Mira, J. (2020). Pandemia COVID-19: Y ahora ¿qué? Journal of Healthcare Quality Research, 35(3), 133-135. https://static.elsevier.es/covid/JHQR_785.pdf.

30. Zhao, G. (2020). Taking preventive measures immediately: evidence from China on COVID-19. Gaceta Sanitaria, 34(3), 217-219. DOI 10.1016/j.gaceta.2020.03.002.

31. Shanafelt, T., Ripp, J., Trockel, M. (2020). Understanding and addressing sources of anxiety among health care professionals during the COVID-19 pandemic. JAMA, 323(21), 2133-2134. https://jamanetwork.com/journals/ jama/fullarticle/2764380.

32. Lima, C. K. T., Carvalho, P. M. M., Lima, I. A. A. S., Nunes, J. U. A. O., Saraiva, J. S. et al. (2020). The emotional impact of Coronavirus 2019-nCoV (new Coronavirus disease). Psychiatry Research, 287, 112915. DOI 10.1016/j. psychres.2020.112915.

33. Talevi, D., Socci, V., Carai, M., Carnaghi, G., Faleri, S. et al. (2020). Mental health outcomes of the COVID-19 pandemic. Rivista di Psichiatria, 55(3), 137-144. DOI 10.1708/3382.33569.

34. Rajkumar, R. P. (2020). COVID-19 and mental health: a review of the existing literature. Asian Journal of Psychiatry, 52, 102066. DOI 10.1016/j.ajp.2020.102066.

35. Da Costa, B. R. C., Pinto, I. (2017). Stress, burnout and coping in health professionals: a literature review. Journal of Psychology and Brain Studies, 1(1), 4, 1-8.

36. Chew, N., Lee, G., Tan, B., Jing, M., Goh, Y. et al. (2020). A multinational, multicentre study on the psychological outcomes and associated physical symptoms amongst healthcare workers during COVID-19 outbreak. Brain, Behavior, and Immunity, S0889-1591(20), 30523-30527. DOI 10.1016/j.bbi.2020.04.049.

37. Li, W., Yang, Y., Liu, Z. H., Zhao, Y. J., Zhang, Q. et al. (2020). Progression of mental health services during the COVID-19 outbreak in China. International Journal of Biological Sciences, 16(10), 1732-1738. DOI 10.7150/ ijbs. 45120 .

38. Tsamakis, K., Rizos, E., Manolis, A., Chaidou, S., Kympouropoulos, S. et al. (2020). COVID-19 pandemic and its impact on mental health of healthcare professionals. Experimental and Therapeutic Medicine, 19, 3451-3453. DOI 10.3892/etm.2020.8646.

39. Raurell-Torredà, M., Martínez-Estalella, G., Frade-Mera, M. J., Carrasco Rodríguez-Rey, L. F., Romero de San Pío, E. (2020). Reflections arising from the COVID-19 pandemic [Reflexiones derivadas de la pandemia COVID-19]. Enfermería Intensiva (English ed.), 31(2), 90-93. DOI 10.1016/j.enfie.2020.03.001.

40. Hall, L. H., Johnson, J., Watt, I., Tsipa, A., O'Connor, D. B. (2016). Healthcare staff wellbeing, burnout, and patient safety: a systematic review. PLoS One, 11(7), e0159015. DOI 10.1371/journal.pone.0159015.

41. Zhang, W. R., Wang, K., Yin, L., Zhao, W. F., Xue, Q. et al. (2020). Mental health and psychosocial problems of medical health workers during the COVID-19 epidemic in China. Psychotherapy and Psychosomatics, 89(4), $242-250$.

42. Burton, C., Fink, P., Henningsen, P., Löwe, B., Rief, W. et al. (2020). Functional somatic disorders: discussion paper for a new common classification for research and clinical use. BMC Medicine, 18(1), 279. DOI 10.1186/ s12916-020-1505-4. 